\title{
Automatic tactile thematic map generation: research agenda and possible solutions
}

\author{
Jakub Wabiński a, *, Albina Mościcka ${ }^{\text {a }}$, \\ ${ }^{a}$ Military University of Technology, JakubWabiński, jakub.wabinski@wat.edu.pl, Albina Mościcka, albina.moscicka@wat.edu.pl \\ * Corresponding author
}

Keywords: Tactile map, automatic map generation, map generalization, generalization algorithm, blind and visually impaired

\begin{abstract}
:
A lot has been done regarding automatic generation of topographic maps within National Mapping Agencies (NMAs) and there are examples of successful implementations of such projects. The main issue related to automatic map production is cartographic generalization. It is mainly used for transforming the original spatial dataset into maps of smaller scale. Everyone, who has ever worked on map generalization knows, how laborious and time-consuming this process is. This is why a lot of effort is being put to automate it. Automatic map production is very difficult but it gets even more complicated if we consider automatic production of tactile maps - maps that are being read with sense of touch and, to a limited extent, also with eyes.

In an average, a man without visual impairment is capable of distinguishing two points as separate if they are, according to different sources, 0.2-0.3 millimetres apart from each other. If one would like to achieve the same but using sense of touch, a distance of 2.4-3.0 millimetres is necessary. This is enough to show how intense the generalization process has to be while transforming scales of tactile maps. It also brings up a question: 'What are the algorithms and solutions for tactile spatial data generalization and to what extent can this process be automated?'. The answer to this question is the main point of the research presented here.
\end{abstract}

During the presentation, the results of a systematic literature review on this topic basing on the primary studies from the last decade, will be presented. Automatic map generation is nothing new but this field of research lacks a systematic review, which would summarize existing literature. This review, although about automatic map generation in general, focuses on tactile maps. Therefore, the answers to the following questions will be presented:

1. What are the generalization methods and models for automatic (tactile) map generation?

2. What are the existing systems and solutions allowing automatic (tactile) map generation?

3. How to properly design spatial database for automatic map generation?

Presented research will form a significant part of Jakub Wabiński PhD dissertation, which main goal is to create a methodology that would allow blind users to create on-demand thematic maps with different level of detail and scales, out of publicly available spatial data. Due to the fact that in European Union there is the INSPIRE Directive (Infrastructure for Spatial Information in the European Community), which requires member countries to provide their citizens with current spatial data, but also aims to define common standards of describing and sharing spatial data - it is possible to create universal methodology for the whole European Union. The problem is that these data have to be first adapted for use by blind and visually impaired people.

There is high demand on tactile maps and atlases but unfortunately their production is very expensive. Not all the schools for blind and visually impaired can afford to buy them (not to mention individual people). Traditional tactile maps production methods, such as 'thermoforming', are cost effective only in the case of production in a large scale. Pretty often individual map sheets are required to present a certain phenomenon. Fortunately, there are cheap and efficient alternatives - namely 3D printing or swell-paper, which can be used at home by individual users with success. We believe that a platform allowing blind and visually impaired to generate easy-to-use, unique thematic and topographic maps that comply with the requirements regarding tactile cartographic signs designs would be highly appreciated. Similar solutions already exist but only in the field of orientation and navigation maps and they have their limitations. Thematic tactile maps are very important to perceive various information that are provided by spatial data and we would like to focus on them in our presentation. 